\section{Complications Following Plastic Surgery in Solid Organ Transplant Recipients: A Descriptive Cohort Study}

\section{Abstract}

Background: In 2012, over 28,000 organ transplants were performed in the United States. Long-term survival of solid organ transplant recipients (SOTRs) has increased dramatically due to advancements in surgical technology and immunosuppressive therapies. As a result, the number of SOTRs seeking plastic and reconstructive surgery is increasing. However, the safety of plastic and reconstructive surgical procedures in SOTRs, a concerning clinical population for postoperative complications due to immunosuppression, is not well characterized.

Objective: Estimate prevalence of complications following plastic and reconstructive surgery in SOTRs.

Design: Descriptive cohort study.

Patients: All SOTRs who underwent surgical procedures performed by the Section of Plastic and Reconstructive Surgery faculty at Yale-New Haven Hospital between 2004 and 2011.

Measurements: Transplant history (organ, reason for transplant, immunotherapy regimens) and plastic surgery history (procedure, results, and complications) were recorded.

Results: 92 plastic surgical procedures were performed on 65 SOTRs in between 2004-2011, including hand, reconstructive and cosmetic cases. 57 procedures were urgent or emergent, while 35 procedures were elective (of which 23 procedures (66\%) were cosmetic). The overall complication prevalence was $23.9 \%$, most commonly related to wound healing complications $(14.1 \%)$, bleeding $(2.2 \%)$ and postoperative pain $(2.2 \%)$. There was one death in the series $(1.1 \%)$. Lower complication prevalence was observed in elective (6\%) and cosmetic (4\%) procedures.

Conclusions: The overall complication prevalence for plastic surgery in SOTRs was $23.9 \%$, with complication prevalence of $6 \%$ in elective cases. Given this prevalence, this report suggests that a history of transplantation is not an absolute contraindication to plastic and reconstructive surgery despite medical immunosuppression. In particular, elective procedures in suitable patients can have successful outcomes with minimal wound healing complications.

Keywords: Solid organ transplantation; Safety; Plastic surgery
Elizabeth Zellner', Rachel Lentz², Carolyn Chuang ${ }^{1}$ and Derek Steinbacher ${ }^{1}$

1 Department of Surgery, Yale University School of Medicine, New Haven, CT, USA

2 Department of Surgery, University of California San Francisco, San Francisco, CA, USA

Corresponding author: Elizabeth Zellner

झ elizabeth.zellner@yale.edu

MD, Chief Resident in Plastic Surgery, Section of Plastic and Reconstructive Surgery, Department of Surgery, Yale University School of Medicine, P.O. Box 208041, New Haven, CT 06520, USA.

Tel: 9105271871

Citation: Zellner E, Lentz R, Carolyn C, et al. Complications Following Plastic Surgery in Solid Organ Transplant Recipients: A Descriptive Cohort Study. J Aesthet Reconstr Surg. 2016, 2:1.

\section{Introduction}

The first successful kidney transplant was performed by Dr. Joseph Murray, a plastic surgeon, in 1952 [1, 2]. Since that time, significant advances in transplant surgery and immunologic research led to longer lives for solid organ transplant recipients (SOTRs). Resultant improved quality of life led to increased demand for plastic and reconstructive surgery in SOTRs. One 
common indication for surgical management in SOTRs is squamous cell carcinoma, which presents more frequently and at more advanced stage in SOTRs [3].

Few studies investigate outcomes of surgeries performed on SOTRs after transplantation. Case reports describe individual instances of surgery performed on SOTRs [4, 5]. Case series assessing safety of plastic surgery in SOTRs, including free flap reconstruction [6], found no definitive contraindication to performing these procedures in carefully selected SOTRs $[6,7]$. In a 2009 nationwide survey, $25 \%$ of responding plastic surgeons reported they performed plastic surgery procedures in SOTRs with an extremely low complication prevalence based on self-report [8]. Despite these previous reports, outcomes data on plastic surgery in SOTRs is still limited. The objective of this study was to examine complication prevalence in plastic surgery procedures by reviewing longitudinal data for all plastic surgery cases in SOTRs performed at Yale-New Haven Hospital in between 2004-2011.

\section{Objectives}

Our objective was to estimate the complication prevalence of plastic surgery procedures in SOTRs at Yale-New Haven Hospital.

\section{Methods}

We used a descriptive cohort design. We identified participants as all adult SOTRs who underwent plastic and reconstructive surgery at Yale-New Haven Hospital from 2004 to 2011. We longitudinally followed their clinical course through 2014 to identify postoperative complications. Transplant, immunosuppression, and plastic surgery history were extracted and verified by authors $\mathrm{RL}$ and EZ via institutional review board-approved retrospective chart review. Follow-up data was available for all participants. Complications were identified by examining provider notes in the electronic medical record and noting any problems reported on provider assessment and whether any interventions were required. Expected complications included pain, bleeding, infection, and poor wound healing.

Study bias was reduced by reporting all known cases at our institution over eight years. Possible sources of bias include loss to follow up and surgeon-specific patient selection among SOTRs desiring or requiring plastic and reconstructive surgery.

The number of cases performed at our institution during the study period determined the sample size. Complication frequency was calculated using simple proportions. Association analysis was performed using chi square tests, and surgery history characteristics were compared using two-sided t-tests. Loss to follow up was not observed in this sample due to frequent medical visits required of SOTRs.

\section{Results}

65 adult SOTRs who underwent 92 distinct plastic and reconstructive procedures were identified. The sample was $55.4 \%$ male, with an average age of 51 years (SD 11 years), and a mean of 8 years (SD 7 years) between transplantation and subsequent plastic surgery procedure (Table 1). There were 45 kidney recipients, 10 kidney and pancreas recipients, 6 liver recipients, 3 heart recipients and 1 pancreas recipient. The 92 procedures represented both elective (35) and non-elective (57) operations representing a wide range of plastic and reconstructive surgery cases (Table 2).

During the acute perioperative period, patients continued their existing immunosuppressive regimens without alterations. No stress dose steroids were given to any patients and no instances of adrenocortical insufficiency occurred.

During the 140 person-years of follow-up (mean 2 years, SD 2 years), the overall observed complication prevalence was $23.9 \%$ $(n=22)$. The majority $(n=15)$ were wound healing complications in the setting of contaminated wounds that improved with further surgical debridement and meticulous wound care. Other complications observed included bleeding $(n=2)$, pain requiring hospital admission ( $n=2)$, respiratory difficulty prolonging hospital stay $(n=2)$ and acute renal graft failure resulting in one patient's death $(n=1)$.

Among elective procedures $(n=35)$, the complication prevalence was $6 \%$. Among the cosmetic procedures $(n=25)$, the complication prevalence was $4 \%$, representing one instance of postoperative pain following a panniculectomy.

There was no statistical difference between uncomplicated and complicated cases in terms of age (51 years vs. 53 years,

Table 165 adult SOTRs who underwent 92 distinct plastic and reconstructive procedures.

\begin{tabular}{|l|c|}
\hline Demographics & No. (\%) \\
\hline Characteristic & 65 \\
\hline Total no. of patients & \\
\hline Sex & $36(55.4)$ \\
\hline Male & $29(44.6)$ \\
\hline Female & - \\
\hline Age, years & 51 \\
\hline Mean & 11 \\
\hline Standard Deviation & \\
\hline Ethnicity & $39(60)$ \\
\hline White & $16(24.6)$ \\
\hline Black & $10(15.4)$ \\
\hline Hispanic & \\
\hline Organ transplanted & $45(69.2)$ \\
\hline Kidney & $10(15.4)$ \\
\hline Kidney and Pancreas & $6(9.2)$ \\
\hline Liver & $3(4.6)$ \\
\hline Heart & $1(1.5)$ \\
\hline Pancreas & \\
\hline Reason for transplantation & $20(30.8)$ \\
\hline Diabetes & $8(12.3)$ \\
\hline Polycystic Kidney Disease & $5(7.7)$ \\
\hline Glomerulonephritis & $4(6.2)$ \\
\hline Hypertension & $3(4.6)$ \\
\hline Hepatitis C Cirrhosis & $25(38.5)$ \\
\hline Other & \\
\hline Time from transplant to plastic surgery, years & 8 \\
\hline Mean & 7 \\
\hline Standard Deviation & \\
\hline
\end{tabular}


Table 292 procedures represented both elective (35) and non-elective (57) operations representing a wide range of plastic and reconstructive surgery cases.

\begin{tabular}{|c|c|c|}
\hline & $\begin{array}{l}\text { Procedures } \\
\text { Performed }\end{array}$ & Complications \\
\hline Total No. of Procedures & 92 & 22 \\
\hline Hand Surgery & $20(22 \%)$ & $4(18 \%)$ \\
\hline Endoscopic carpal tunnel release & 3 & - \\
\hline Incision and drainage & 3 & - \\
\hline Arthroplasty/Arthrodesis & 2 & - \\
\hline Mass excision & 4 & - \\
\hline Trigger finger release & 2 & - \\
\hline Closed reduction and fixation & 2 & - \\
\hline Open Reduction and fixation & 1 & - \\
\hline Tophus excision & 1 & - \\
\hline DIP pinning & 1 & - \\
\hline Vascular bypass brachial a to palmar arch & 1 & - \\
\hline Breast Surgery & $14(15 \%)$ & $3(14 \%)$ \\
\hline Minor Revision-Reconstructed Breast & 6 & - \\
\hline Reduction Mammoplasty & 5 & - \\
\hline Free Flap Reconstruction & 2 & - \\
\hline Implant Reconstruction & 1 & - \\
\hline Trunk Surgery & $26(28 \%)$ & $7(32 \%)$ \\
\hline VHR-Component Separation & 6 & - \\
\hline Wound Debridement & 5 & - \\
\hline Panniculectomy & 5 & - \\
\hline VHR-Mesh & 4 & - \\
\hline Mass excision & 4 & - \\
\hline Wound Revision and Local Flap & 1 & - \\
\hline Pressure Ulcer with Muscle Flap & 1 & - \\
\hline Lower Extremity & $11(12 \%)$ & $6(27 \%)$ \\
\hline Debridement with STSG & 5 & - \\
\hline Debridement with local flap & 2 & - \\
\hline Debridement alone & 2 & - \\
\hline Mass excision & 2 & - \\
\hline Head and Neck & $21(23 \%)$ & $2(9 \%)$ \\
\hline Mass excision with local flap & 8 & - \\
\hline
\end{tabular}

$p=0.38)$, gender $(54 \%$ male vs. $32 \%$ male $p=0.06)$, race, or preoperative creatinine clearance ( 2.0 vs. $2.2, p=0.77$ ). Patients with complications were more likely to have diabetes mellitus than those without ( $48 \%$ vs. $21 \%$ ).

A chi-square test for association was conducted between immunosuppressant medication type (mycophenolate, cyclosporine, sirolimus, tacrolimus, azathioprine, and prednisone) and complication occurrence. There were no statistically significant associations between any particular immunosuppressant medication and complication occurrence.

It was noted that while complications in hand, breast and trunk surgery reflected case prevalence, a higher rate of complications were noted in lower extremity cases and a lower rate of complications were noted in head and neck procedures (Table 3 ). There was a statistically significant difference on two-sided t-test of procedure time between the complicated and uncomplicated cases. The complicated cases had an average duration of 168 minutes while the uncomplicated cases had an average duration of 70 minutes ( $p=0.03$ ) (Tables 1 and 2).

\section{Discussion}

Complication prevalence for plastic surgery procedures in SOTRs was $23.9 \%$, the majority being wound healing complications. As noted in previous papers, early, aggressive debridement and broad spectrum antibiotics are advocated in these patients to lower the risk of wound healing complications [9]. SOTRs are at higher risk of poor wound healing and infection due to immunosuppression and other comorbid factors, and previous reports displayed complication prevalence of $28-52 \%$, also predominated by wound healing/infection [10].

Complications more frequently occurred in urgent/emergent cases compared to elective cases ( $35 \%$ vs. $6 \%$, respectively). This association may result from various factors including existing contaminated wound and nonoptimal medical condition at time of surgery. Lower extremity cases were also more associated with complications, while head and neck cases were less likely to result in complications, possibly a reflection of blood supply in patients who were often affected by diabetes mellitus and vascular conditions such as hypertension. Additionally, longer procedures were significantly more likely to have complications. Lengthier procedures may represent increased morbidity of the surgical indication, increased surgical difficulty, and increased physiologic stressors on the patient.

SOTRs undergoing cosmetic surgery experienced particularly low complication prevalence, with only one complication (postoperative pain) in 25 patients. This low complication prevalence suggests that cosmetic surgery can safely be performed in SOTRs with other comorbidities. Through direct interaction, these patients expressed great satisfaction with their final outcomes and quality of life improvements. We believe that low complication prevalence shows that careful patient selection in this population can lead to successful outcomes for SOTRs seeking elective and cosmetic surgery.

Transplant recipients have an increased risk of cutaneous malignancy secondary to their lifelong immunosuppressive status [3]. Therefore, their need for plastic and reconstructive surgery is higher than the general population. In our study, 22 patients presented to plastic surgeons because of skin malignancies. Among these patients undergoing relatively simple soft tissue excisions with local flap coverage, there were no complications.

We saw no significant association of wound healing complications with any particular immunosuppressant. A larger sample size is indicated to further investigate the interactions of these complex, long-term medications. Previous work outlined the theoretical impedance of wound healing by immunomodulating medications [6]. However, when patients did not present with existing infection, wound healing complication prevalence was in line with the general population.

There was one death in the series in a 69-year-old kidney transplant patient who underwent a complex ventral hernia repair 
Table 3 Description of complications and corresponding patient characteristics.

\begin{tabular}{|c|c|c|c|c|c|c|c|c|}
\hline $\begin{array}{l}\text { Age } \\
\text { (yr) }\end{array}$ & $\begin{array}{l}\text { Type of } \\
\text { Transplant }\end{array}$ & Cause of Txp & $\begin{array}{l}\text { Elective/ } \\
\text { Cosmetic? }\end{array}$ & Immunosupp & Problem & Surgery & Complication & Outcome \\
\hline 61 & Kidney-DDKT & DM & & $\begin{array}{l}\text { MMF, siro, } \\
\text { pred }\end{array}$ & Necrotic fingertip & $\begin{array}{l}\text { Amputation L ring } \\
\text { finger with local flap } \\
\text { coverage }\end{array}$ & $\begin{array}{l}\text { Necrosis of } \\
\text { fingertip }\end{array}$ & $\begin{array}{c}\text { Revision } \\
\text { amputation/flap } \\
\text { coverage }\end{array}$ \\
\hline 41 & Kidney-DDKT & \multicolumn{2}{|c|}{ IgA Nephropathy } & MMF, tac, pred & $\begin{array}{l}\text { Scapholunate } \\
\text { ligament rupture }\end{array}$ & $\begin{array}{l}\text { Scapholunate } \\
\text { arthrodesis }\end{array}$ & $\begin{array}{l}\text { Pain, eventual } \\
\text { scapholunate } \\
\text { collapse }\end{array}$ & $\begin{array}{l}\text { Revision } \\
\text { scapholunate } \\
\text { arthrodesis }\end{array}$ \\
\hline 35 & Kidney-DDKT & DM & & MMF, tac, pred & $\begin{array}{l}\text { Chronic L upper } \\
\text { extremity ischemia }\end{array}$ & $\begin{array}{l}\text { L vascular bypass } \\
\text { brachial a to palmar } \\
\text { arch }\end{array}$ & Skin necrosis & Debridement \\
\hline 36 & Kidney-DDKT & DM & & MMF, tac, pred & Open wound L hand & $\begin{array}{l}\text { L Hand debridement } \\
\text { and groin flap }\end{array}$ & $\begin{array}{l}\text { Prolonged healing } \\
\text { of skin graft }\end{array}$ & $\begin{array}{c}\text { Weekly } \\
\text { debridements }\end{array}$ \\
\hline 48 & $\begin{array}{l}\text { Kidney-Living } \\
\text { unrelated }\end{array}$ & DM & & MMF, tac, pred & $\begin{array}{c}\text { Necrotic abdominal } \\
\text { wound }\end{array}$ & $\begin{array}{c}\text { Debridement of } \\
\text { abdominal wound/ } \\
\text { washout }\end{array}$ & $\begin{array}{l}\text { Poor wound } \\
\text { healing, persistent } \\
\text { open pannus } \\
\text { wound }\end{array}$ & $\begin{array}{l}\text { Hospitalized, } \\
\text { repeat I and D, } \\
\text { antibiotics }\end{array}$ \\
\hline 60 & $\begin{array}{l}\text { Kidney- } \\
\text { Unspecified }\end{array}$ & DM & & MMF, tac, pred & $\begin{array}{l}\text { R heel wound/ } \\
\text { calcaneal } \\
\text { osteomyelitis }\end{array}$ & $\begin{array}{c}\text { STSG from L } \\
\text { anterolateral thigh }\end{array}$ & $\begin{array}{c}\text { poor wound } \\
\text { healing to STSG }\end{array}$ & N/A \\
\hline 51 & $\begin{array}{l}\text { Kidney and } \\
\text { Pancreas-DD }\end{array}$ & DM & & MMF, tac, pred & $\begin{array}{l}\text { Fasciotomy wounds } \\
\text { (2/2 compression } \\
\text { syndrome) }\end{array}$ & $\begin{array}{l}\text { STSG and complex } \\
\text { local closure }\end{array}$ & $\begin{array}{l}\text { Unable to salvage } \\
\text { leg }\end{array}$ & BKA \\
\hline 52 & $\begin{array}{l}\text { Kidney-Living } \\
\text { related }\end{array}$ & \multicolumn{2}{|c|}{ Glomerulonephritis } & $\begin{array}{l}\text { MMF, siro, } \\
\text { pred }\end{array}$ & $\begin{array}{c}\text { Suture abscess } R \\
\text { abdomen }\end{array}$ & Excision/Debridement & $\begin{array}{l}\text { Persistent draining } \\
\text { wound }\end{array}$ & Repeat I and D \\
\hline 53 & $\begin{array}{l}\text { Kidney and } \\
\text { Pancreas-DD }\end{array}$ & DM & & Tac, pred & $\begin{array}{c}\text { Nonhealing L heel } \\
\text { ulcer }\end{array}$ & Sural island flap c STSG & $\begin{array}{l}\text { Infection/partial } \\
\text { flap loss }\end{array}$ & $\begin{array}{c}\text { Multiple } \\
\text { debridements }\end{array}$ \\
\hline 50 & $\begin{array}{l}\text { Kidney and } \\
\text { Pancreas-DD }\end{array}$ & DM & & $\begin{array}{l}\text { CSA, pred, } \\
\text { MMF }\end{array}$ & $\begin{array}{l}\text { R foot plantar } \\
\text { wound }\end{array}$ & Debridement/STSG & $\begin{array}{c}\text { Cellulitis/ } \\
\text { osteomyelitis }\end{array}$ & $\begin{array}{c}\text { Multiple } \\
\text { debridements, } \\
\text { eventual STSG }\end{array}$ \\
\hline 52 & $\begin{array}{c}\text { Kidney and } \\
\text { Pancreas-DD }\end{array}$ & DM & & $\begin{array}{l}\text { CSA, pred, } \\
\text { MMF }\end{array}$ & $\begin{array}{l}\text { Persistent foot } \\
\text { osteomyelitis }\end{array}$ & Debridement/STSG & $\begin{array}{l}\text { Poor wound } \\
\text { healing, chronic } \\
\text { osteomyelitis }\end{array}$ & SYME amputation \\
\hline 46 & Heart & \multicolumn{2}{|c|}{$\begin{array}{l}\text { Nonischemic } \\
\text { cardiomyopathy }\end{array}$} & CSA, MMF & S/P Mastectomy & Bilateral TRAM & $\begin{array}{l}\text { fat necrosis/ } \\
\text { ventral hernia }\end{array}$ & $\begin{array}{c}\text { TRAM revisions/ } \\
\text { VHR }\end{array}$ \\
\hline 53 & $\begin{array}{c}\text { Kidney-DDKT } \\
\times 2\end{array}$ & DM & & MMF, tac, pred & $\begin{array}{c}\text { Deflation/ } \\
\text { discomfort of tissue } \\
\text { expanders }\end{array}$ & $\begin{array}{l}\text { Removal Bilateral } \\
\text { Tissue Expanders }\end{array}$ & Post op hypoxia & $\begin{array}{c}\text { Hospitalized } \times 2 \\
\text { days }\end{array}$ \\
\hline 50 & $\begin{array}{l}\text { Kidney and } \\
\text { Pancreas-DD }\end{array}$ & DM & & $\begin{array}{l}\text { CSA, pred, } \\
\text { MMF }\end{array}$ & S/P Mastectomy & $\begin{array}{l}\text { Tissue expander } \\
\text { placement }\end{array}$ & $\begin{array}{c}\text { Bilateral } \\
\text { Hematomas }\end{array}$ & $\begin{array}{l}\text { Hematoma } \\
\text { evacuation }\end{array}$ \\
\hline 62 & Heart & \multicolumn{2}{|c|}{ Ischemic cardiomyopathy } & siro, MMF & $\begin{array}{l}\text { Massive abdominal } \\
\text { incisional hernia }\end{array}$ & Autologous repair & Wound infection & $\begin{array}{l}\text { Debridement/ } \\
\text { VAC placement }\end{array}$ \\
\hline 47 & $\begin{array}{l}\text { Kidney-Living } \\
\text { related }\end{array}$ & PCKD & & $\begin{array}{l}\text { MMF, siro, } \\
\text { pred }\end{array}$ & $\begin{array}{l}\text { recurrent ventral } \\
\text { hernia }\end{array}$ & $\begin{array}{l}\text { VHR comp separation } \\
\text { and mesh }\end{array}$ & $\begin{array}{c}\text { necrotic } \\
\text { abdominal wound }\end{array}$ & $\begin{array}{l}\text { debridement/ } \\
\text { removal of mesh }\end{array}$ \\
\hline 61 & Kidney-DDKT & DM & & CSA, pred & $\begin{array}{l}\text { recurrent ventral } \\
\text { hernia }\end{array}$ & VHR comp separation & aspiration PNA & $\begin{array}{c}\text { recurrent hernia, } \\
\text { laparoscopic } \\
\text { repair }\end{array}$ \\
\hline 69 & Kidney-DDKT & PCKD & Elective & Pred & $\begin{array}{l}\text { ventral/umbilical } \\
\text { hernia }\end{array}$ & $\begin{array}{l}\text { Hernia repair with } \\
\text { alloderm }\end{array}$ & $\begin{array}{l}\text { ARF, Abdominal } \\
\text { compartment } \\
\text { syndrome } 2 / 2 \\
\text { mesenteric } \\
\text { ischemia }\end{array}$ & $\begin{array}{l}\text { SICU admission, } \\
\text { compartment } \\
\text { syndrome, ex-lap, } \\
\text { expired during } \\
\text { admission }\end{array}$ \\
\hline 59 & Liver & $\mathrm{HCV}$ & & $\begin{array}{l}\text { MMF, siro, } \\
\text { pred }\end{array}$ & BCC nose & $\begin{array}{l}\text { Excision and repair, } \\
\text { recon with local flaps } \\
\text { and free cartilage graft }\end{array}$ & $\begin{array}{l}\text { Wound healing } \\
\text { difficulty, partial } \\
\text { loss of alar rim }\end{array}$ & $\begin{array}{l}\text { Nasal flap } \\
\text { revision }\end{array}$ \\
\hline
\end{tabular}




\begin{tabular}{|c|c|c|c|c|c|c|c|c|}
\hline 64 & Heart & \multicolumn{2}{|c|}{ Ischemic cardiomyopathy } & CSA, MMF & $\begin{array}{l}\text { SCC } L \text { ear, } R \text { ear, } L \\
\text { cheek } L \text { forehead }\end{array}$ & $\begin{array}{l}\text { FTSG R ear, Flap L ear, } \\
\text { FTSG L forehead, RFFF } \\
\text { to } L \text { cheek }\end{array}$ & Bleeding & $\begin{array}{c}\text { OR exploration, } \\
\text { intubation/SICU } x \\
3 \text { weeks }\end{array}$ \\
\hline 66 & $\begin{array}{c}\text { Kidney-Living } \\
\text { unrelated }\end{array}$ & \multicolumn{2}{|c|}{$\begin{array}{c}\text { Congenital hypoplastic } \\
\text { kidney }\end{array}$} & MMF, tac, pred & $\begin{array}{l}\text { Anterior thigh } \\
\text { defect } 2 / 2 \text { SCC } \\
\text { excision }\end{array}$ & $\begin{array}{l}\text { Advancement flap, } \\
\text { SLNBX }\end{array}$ & $\begin{array}{c}\text { Persistent } \\
\text { indurated groin } \\
\text { mass }\end{array}$ & Surgical excision \\
\hline 41 & Liver $\times 2$ & $\begin{array}{c}\text { Autoimmune } \\
\text { hepatitis }\end{array}$ & $\begin{array}{l}\text { Elective, } \\
\text { Cosmetic }\end{array}$ & Tac, pred & Abdominal pannus & $\begin{array}{l}\text { Upper abdominal } \\
\text { panniculectomy }\end{array}$ & Uncontrolled pain & $\begin{array}{l}\text { Hospital } \\
\text { admission }\end{array}$ \\
\hline
\end{tabular}

DD: Deceased Donor; DDKT: Deceased Donor Kidney Transplant; MMF: Mycophenylate Mofetil; pred: Prednisone; tac: Tarolimus; CSA: Cyclosporine A; BCC: Basal Cell Carcinoma; SCC: Squamous Cell Carcinoma; STSG: Split Thickness Skin Graft; TRAM: Transverse Rectus Abdominis Muscle; FTSG: Full Thickness Skin Graft; VHR: Ventral Hernia Repair; RFFF: Radial Forearm Free Flap; SLNBX: Sentinel Lymph Node Biopsy; BKA: Below Knee Amputation

in conjunction with the general surgery team. Perioperatively, the patient developed acute renal failure and mesenteric ischemia, thought to be due to transient hypovolemia during the case. The patient went on to develop abdominal compartment syndrome and underwent an exploratory laparotomy by general surgery. Unfortunately, he ultimately expired 5 days after the original hernia repair. Although this outcome represents the ultimate fear of a plastic surgeon performing an elective case on a complex patient, we do not believe it negates the many other successful outcomes in SOTRs. Rather, it demonstrates the importance of careful hemodynamic monitoring with these complex patients and the close cooperation required between multiple surgical teams and the anesthesia and critical care teams during these cases.

Limitations to this study include surgeon-specific patient selection, reliance on medical records for source of data, and inclusion of only procedures performed by plastic surgery faculty at an academic center. Patient selection factors underestimate potential complication prevalence of surgery in SOTRs, since patients recommended to undergo surgery may have relatively few comorbid conditions. Medical records may have inaccurate or incomplete reporting of information. Procedures performed by plastic surgery faculty at an academic tertiary care center may lead to different complication prevalence compared to other practice settings.

The population assessed is representative of SOTRs at an academic tertiary care center. We reported our cohort's transplant history and plastic surgery case mix for comparison to other populations. It is important to note that at our institution, there is a robust interdisciplinary transplant surgery program that oversees the care of all patients with a history of solid organ transplantation. The transplant surgery service works closely with medical teams and surgical subspecialty teams to optimize the care of these patients (Figure 1).

As the incidence of solid organ transplantation continues to rise, an increasing number of transplant recipients require plastic and reconstructive surgeons $[7,11,12]$. Some guidelines exist for surgical intervention in SOTRs, though not specifically in the field

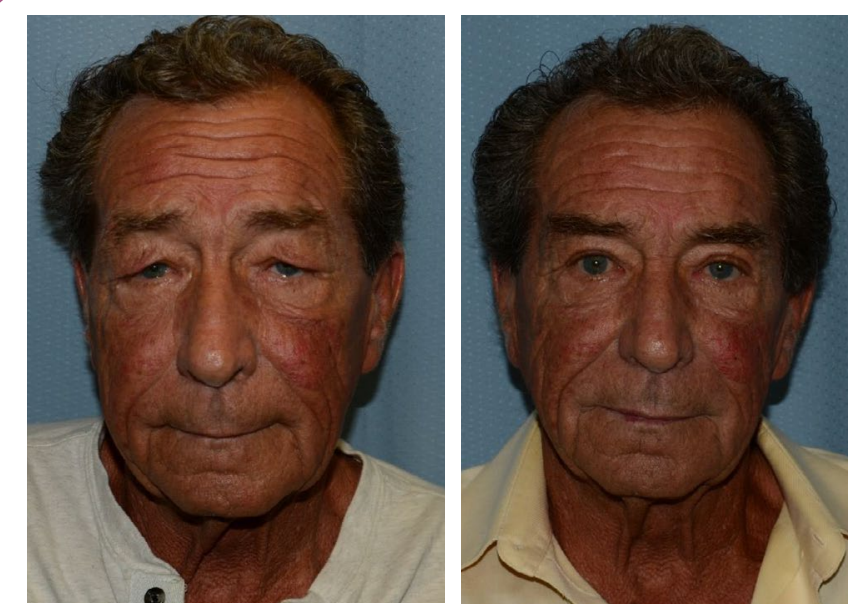

Figure 1 A 67 Year old male patient who underwent bilateral blepharoplasty with significant improvement in vision and cosmesis.

of plastic surgery [13-15]. In addition, many SOTRs on current immunosuppressive regimens go on to lead relatively normal lives and would like to have access to elective plastic surgery procedures. As this demand expands, an assessment of ongoing surgical safety and outcomes in this potentially morbid patient population is warranted.

\section{Conclusion}

As solid organ transplantation becomes increasingly common, plastic surgeons will have more interactions with transplant patients in both emergent and elective settings. This study estimates an overall plastic surgery complication prevalence of $23.9 \%$ in SOTRs for all indications, with lower complication prevalence in elective and cosmetic cases $16 \%$ and $4 \%$ respectively). This provides insight on complication risk when assessing the risk-benefit ratio of pursuing plastic surgery procedures in the immunosuppressed SOTR. It also restates the importance of early intervention and adequate debridement to avoid wound complications in cases requiring urgent attention. The STROBE guidelines were followed in the preparation of this manuscript $[16,17]$. 


\section{References}

1 UNOS (2013) Transplants by Donor Type.

2 Murray JE, Merrill JP, Harrison H (1958) Kidney Tranplantation Between Seven Pairs of Identical Twins. Annals of Surgery 148: 343-357.

3 Berg D, Otley CC (2002) Skin cancer in organ transplant recipients: Epidemiology, pathogenesis and management. Journal of American Academic Dermatology 47: 1-20.

4 Cizmeci O, Ozden BC (2006) Abdominoplasty in a Liver Transplant Patient. Plastic and Reconstructive Surgery 117: 1069-1070.

5 Peled ZM, Cooper JS, Cooper RN (2008) Aesthetic Surgery in the Transplant Patient: Pushing the Envelope? Plastic and Reconstructive Surgery 122: 69e-70e.

6 Lee AB, Dupin CL, Colen L, Jones NF, May JW, et al., (2007) Microvascular Free Tissue Transfer in Organ Transplantation Patients: Is It Safe? Plastic and Reconstructive Surgery 121: 1986-1992.

7 Cohen M, Pollak R, Garcia J, Mozes MF (1989) Reconstructive Surgery for Immunosuppressed Organ-Transplant Recipients. Plastic and Reconstructive Surgery 83: 291-295.

8 Sweis I, Tzvetanov I, Benedetti E (2009) The New Face of Transplant Surgery: A Survery on Cosmetic Surgery in Transplant Recipients. Aesthetic Plastic Surgery 33: 819-826.

9 Klein MB, Chang J (2000) Management of Hand and Upper-Extremity Infections in Heart Transplant Recipients. Plastic and Reconstructive Surgery 106: 598-601.
10 Kuppahally S, Al-Khaldi A, Weisshaar D, Valantine HA, Oyer P, et al., (2006) Wound healing complications with de novo sirolimus versus mycophenolate mofetil-based regimen in cardiac transplant recipients. Am J Transplant 6: 986-992.

11 Fa-zhi QI, Yong Z, Zhen Y, Zi-hao F, Jian-ying GU (2009) Plastic surgery after solid organ transplantation. Chinese Medical Journal 122: 1184-1187.

12 Wendt JR, Ulich T, Rao PN (2004) Long-Term Survival of Human Skin Allografts in Patients wtih Immunosuppression. Plastic and Reconstructive Surgery 113: 1347-1354.

13 Testa G, Goldstein RM, Toughanipour A, Abbasoglu O, Jeyarajah D, et al., (1998) Guidelines for Surgical Procedures after Liver Transplantation. Annals of Surgery 227: 590-599.

14 Bakkaloglu M, Hamilton DNH, Macpherson SG, Briggs JD (1978) Morbidity and mortality in renal transplant patients after incidental surgery. British Journal of Surgery 65: 228-230.

15 Papadopoulos O, Konofaos P, Chrisostomidis C, Lionaki S, Georgiou P (2005) Reconstructive Surgery for Kidney Transplant Recipients. Transplantation Proceedings 37: 4218-4222.

16 Elm EV, Altman DG, Egger M, Pocock SJ, Gotzsche PC, et al., (2007) The Strengthening the Reporting of Observational Studies in Epidemiology (STROBE) statement: guidelines for reporting observational studies. Ann Intern Med 335: 806-808.

17 Vandenbroucke JP, Elm EV, Altman DG, Gøtzsche PC, Mulrow CD, et al., (2007) Strengthening the Reporting of Observational Studies in Epidemiology (STROBE): explanation and elaboration. Ann Intern Med 147: W163-W194. 